\title{
A New Less-Loading Extrusion Technology of Mg Alloy Tube Workpiece
}

\author{
Qiang Wang ${ }^{1, a}$, Zhimin Zhang ${ }^{2, b}$, Yong Xue ${ }^{1}$,Jianmin $\mathrm{Yu}^{2}$ \\ ${ }^{1}$ Dept. of Materials Science and Engineering, North University of China, Taiyuan 030051, China \\ ${ }^{2}$ Engineering Research Center of Magnesium-base Material Processing Technology, Ministry of \\ Education, Taiyuan 030051, China
}

awangqiang@nuc.edu.cn, b'zhangzhimin@nuc.edu.cn

\begin{abstract}
Keywords: Magnesium alloy,Backward extrusion,Hollow billet,Pre-deformation,Tube workpiece Abstract. According to the exist problem in process\&manufacturing of large-diameter magnesium alloy tube workpieces, pre-deformation of as-cast alloy and extrusion from hollow billet were developed. The hot compression experiment results show that pre-deformation of as-cast alloy is of advantage to decrease flow stress and to refine grain. The analytic results by slab method and numerical simulation show that forming force is decreased during extrusion from hollow billet because of the decrease of contact area and average positive stress on interface. The forming technology developed was validated and applied. The AZ80 alloy tube workpieces with outside diameter of $550,750 \mathrm{~mm}$ and wall thickness of $35 \mathrm{~mm}$ were extruded on $12.5 \mathrm{MN}$ and $30 \mathrm{MN}$ vertical hydraulic press respectively. The forming forces are reduced and the product performance are improved. It offers a new approach for forming large-diameter tube workpiece of magnesium alloy.
\end{abstract}

\section{Introduction}

Magnesium alloys are promising structural light metals because their low densities, good recyclical potential and abundant resources, which is expected to become a next-generation material [1 4]. This material is receiving special attention for an alternative material to reduce equipment weight because they are 33\% lighter than the weight of aluminum alloys[5]. Magnesium alloys are produced and used in many forms such as casting, sheet, plate, bar, rod, channels and forgings in the automobile, aerospace and electronics industries. Compared to other forming processes, such as forging, or die-casting, extrusion processes are more cost-effective methods for mass-producing tubes or sheets.

Nowadays, the extrusion process has a great important role in the manufacturing industries. Among the different conventional deformation processing methods, the backward extrusion (BE) has become one of the most promising manufacturing processes due to its material savings and high surface quality [6,7]. However,the higher process load is an important limitation of the conventional backward extrusion process. To improve some problems of conventional backward extrusion process: the requirement of large forming machine, the difficulty in choosing the die material caused by high surface pressure, high cost of forming machine due to the improvement of noise and vibration, etc, numerous researches[8,9] had been conducted on reducing extrusion force. V.Shatermashhadi et al. [10] have proposed a new method of backward extrusion using small diameter billet. The load is reduced to about less than a quarter in comparison with the conventional backward extrusion process, which is due to reduce of the cross section of the initial billet. KIM and PARK [11] studied torsional backward extrusion process by using the upper bound technique and FEM simulation. The results revealed that the backward extrusion with low die rotation is a very efficient method because this process generates the lower forming load and high homogeneous deformations.

In this study, a new backward extrusion method for large-diameter tube workpiece of magnesium alloy, consisting of as-cast billet pre-straining followed by extrusion from hollow billet is proposed. The description of this novel less-loading process emphasizing the peak flow stress, the average positive stress on interface and the results of the experiments performed to evaluate the potential for reducing extrusion force are presented. 


\section{As-cast billet pre-deformation}

The pre-strained material is attained by as-cast billet upsetting with the reduction in height of $50 \%$ at $300^{\circ} \mathrm{C}$ (hereafter, denoted pre-strained material). Specimens with a length of $15 \mathrm{~mm}$ and $10 \mathrm{~mm}$ in diameter of as-cast and pre-strained AZ80 magnesium alloy (in weight percent) of 8.43Al, 0.6Zn, and $0.19 \mathrm{Mn}$ were deformed respectively in compression on Gleeble 3800 to investigate the effect of initial grain on hot working stresses. The experiment temperature was ranged from $200-400^{\circ} \mathrm{C}$ and various strain rates 0.01 and $1.0 \mathrm{~s}^{-1}$ under maximum strain of $60 \%$.

The true stress-strain curves in Fig. 1 show that the initial grain has significant effect on flow stress except that strain rate and temperature. At the same deformation conditions, the flow stresses of pre-strained alloy with finer grain are lower than those of as-cast alloy, i.e the flow stress increases with increasing grain size. The effect diminishes with increasing strain and increasing temperature. It is also evident that the strain to the peak stress also increases with increasing grain size. The peak flow stresses of pre-strained alloy are lower 20\% than those of as-cast alloy, as shown in Fig.2. The results are same with M.R.Barnett's investigation for AZ31 alloy [12]. This is indicated of initial grain size influence proceeding of work hardening and dynamic recrystallization during hot compression.

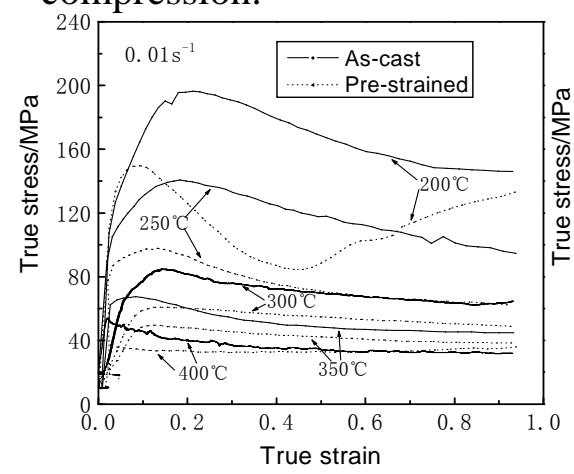

Fig.1 The true stress-strain curve of AZ80 alloy

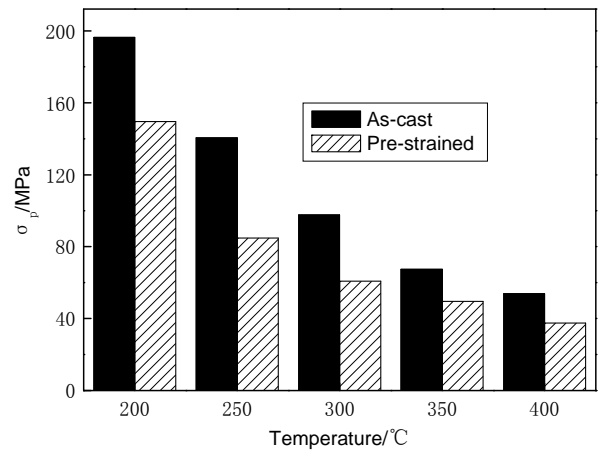

Fig.2 Comparison of peak stress

Actually,finer initial grain obtained by pre-straining can reduce hot working stresses of $\mathrm{Mg}$ alloy, it is also advantageous to improve formability and strengthening effect for subsequent deformation [13].The method of reducing forming force using as-cast billet pre-straining was put forward for magnesium alloy extrusion. In this, pre-straining is given for as-cast magnesium alloy without restraining metal flow and then extrusion is attained with a low flow stress.

\section{Extrusion from hollow billet}

The maximum load was chosen as the extrusion load during extrusion process. A new concept of hollow billet is proposed and a method of extrusion from hollow billet is developed. The schematic diagram of this process is shown in Fig.3. As shown in this figure, a moving cylindrical shaped mandrel with diameter $d_{3}$ is attached to the punch. An initially hollow billet, prepared by as-cast billet upsetting followed by punching, is considered. Its outer and inner diameters are $\mathrm{d}_{1}$ and $\mathrm{d}_{2}$, respectively. At the same time, the extrusion process is used in forming the parts with smaller center hole at the bottom by subsequent upsetting.

After the hollow billet is initially placed into the die cavity, the moving mandrel inserts the hollow billet and then with the pressure of the punch, the material is compressed and flowed into the gap between the punch and the die. The contact area is clearly reduced in comparison with the conventional backward extrusion process. According to the forming principle, extrusion pressure is composed of two parts, one part forces the metal to flow into the annular space between punch and die, the other part upsetting cylindrical metal. Forming force was analyzed by slab method. Fig.4 reveals the normal stress distribution on the contact surface for upsetting part. As shown in Figure 4, it is linear at normal friction condition.Compared with the conventional backward extrusion process, the middle high stress area in the diameter $\mathrm{d}_{3}$ region is removed. Accordingly, the average positive stress on interface reduces during extrusion from hollow billet. 

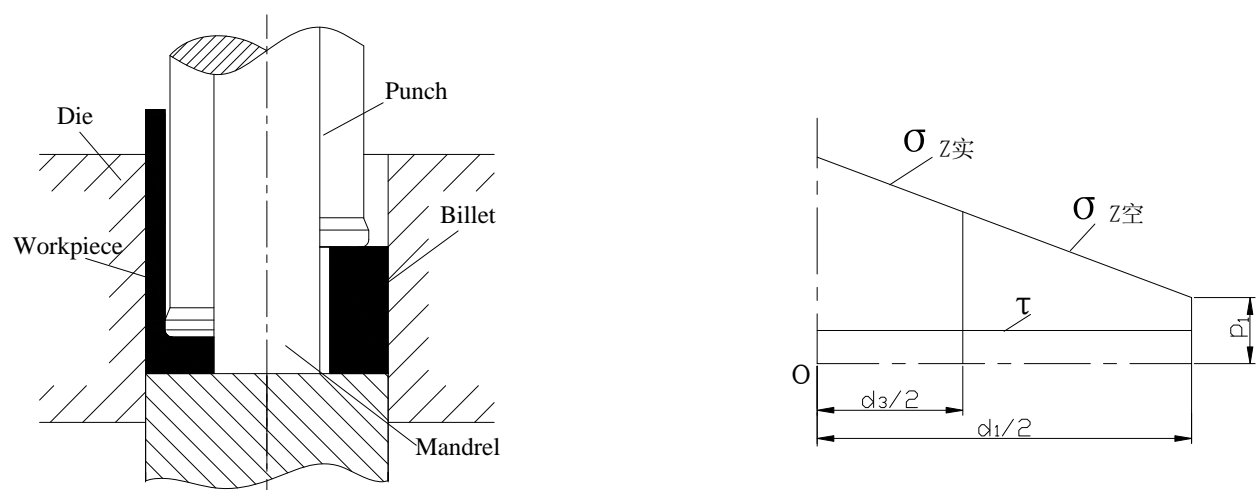

Fig.3 Schematic diagram of new technology Fig.4 Schematic diagram of stress distribution

To investigate the press force during extrusion from hollow billet, the finite element simulation is conducted using an implicit FE code MSC/SuperForm. The data on AZ80 alloy flow stress as function of strain, strain rate and temperature established based on above compression experiment have to be introduced into the FE package. The outer and inner diameters of tube workpiece were set as 100 and $90 \mathrm{~mm}$, respectively. The inner diameters of hollow billet is $60 \mathrm{~mm}$. Fig.5 presents the calculational force-displacement curve during extrusion from hollow billet at different r1 value, i.e. with different diameter mandrel. The results showed that forming force is decreased enormously during extrusion from hollow billet, not only because of the decrease of contact area and also the decrease of average positive stress on interface. When $\mathrm{r} 1$ is 0.6 , the forming force is reduced about less than $40 \%$ in comparison with $r 1=0$, i.e. the conventional backward extrusion process.

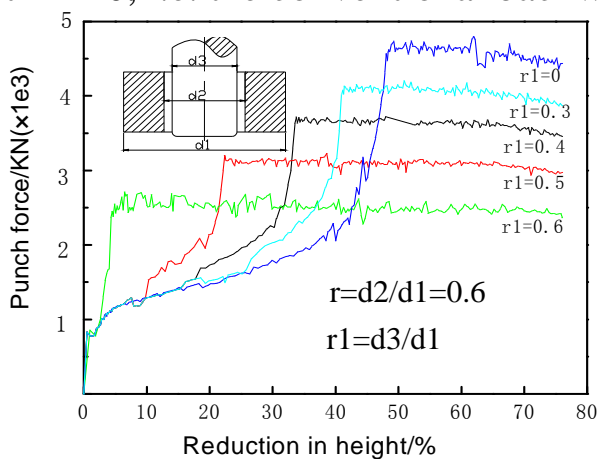

Fig.5 The calculational force-displacement curve

\section{Experiment and results}

According to the above research results, the extrusion technique has been developed for large diameter magnesium alloy tube workpiece. A hollow billet is prepared by upsetting and punching from as-cast alloy rod. And then tube workpiece is extruded from hollow billet. The metal may flow at lower flow stress, smaller cross section of the initial billet and lower average positive stress, which reduces extrusion force. To validate the technological feasibility and to obtain a sound product with an appropriate forming condition, AZ80 alloy extrusion experiments for two kinds of specifications tube workpiece were conducted. The outer diameters of the tube workpiece are 550 and $750 \mathrm{~mm}$, respectively. The wall thickness of the tube workpiece is $35 \mathrm{~mm}$. Accordingly, the inner diameters of the billet were set as 400 and $560 \mathrm{~mm}$, the mandrel diameters were set as 395 and $550 \mathrm{~mm}$ respectively.

The AZ80 alloy was produced by continuous casting method. The rods have been machined in order to remove the effect of the surface layer and homogenized $\left(385^{\circ} \mathrm{C} / 12 \mathrm{~h}\right)$ before deformation(Fig.6a). The hollow billet prepared is shown in Fig.6b. Extrusion were conducted under isothermal conditions in which the die temperatures were varied depending on the billet temperatures. The billets were heated to a temperature within 350 to $380^{\circ} \mathrm{C}$ during forming process. The equipment employed was oil-hydraulic press of capacity 12.5MN, 30MN and average forming speed of a ram $10 \mathrm{~mm} / \mathrm{s}$. A graphite coating was used to lubricate billet and punch surface. 
The magnesium alloy tube workpieces are produced according to the present technique, as shown in Fig.6(c). The forming force was decreased by extrusion from hollow billet. The outer diameters $550 \mathrm{~mm}$ and $750 \mathrm{~mm}$ tube workpiece with wall thickness $35 \mathrm{~mm}$ were extruded on the 12.5MN, 30.0MN oil-hydraulic press respectively. The technology and dies designed have many other advantages such as high production precision, low surface roughness and convenient mould unloading for near-net shape forming. The manufacture costs are reduced due to using limited capacity of press equipment and less forming process.
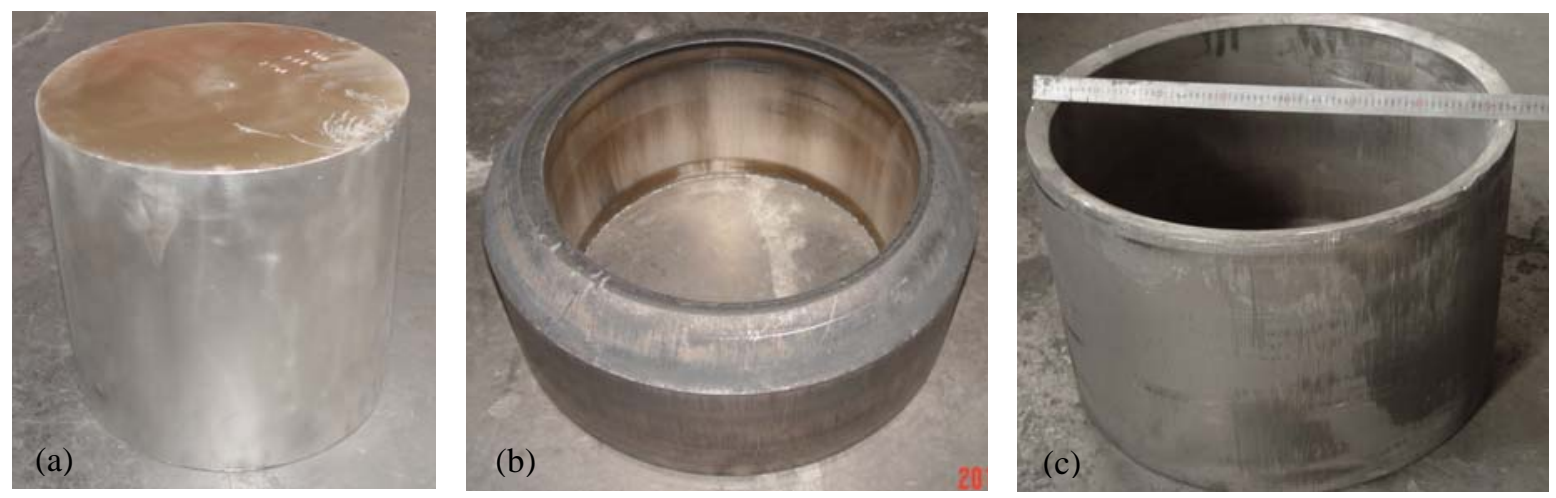

Fig.6 (a) and (b) The BE workpiece before processing, (c) after BE processing

\section{Summary}

According to hot compression results, as-cast billet prestraining is proposed in order to reduce the maximum forming load, in which extrusion is attained with a low flow stress. At the same time formability and mechanical performance are improved for subsequent deformation.

The analytic results by slab method and FEA indicate that extrusion from hollow billet can reduce the maximum forming load because of the decrease of contact area and average positive stress on interface.

By means of the developed technique, the large-diameter tube workpieces with outer diameters $550 \mathrm{~mm}$ and $750 \mathrm{~mm}$ were extruded on the $12.5 \mathrm{MN}, 30.0 \mathrm{MN}$ oil-hydraulic press respectively.

\section{Acknowledgements}

The authors are grateful for the research support of the Program for New Century Excellent Talents in University (NCET-13-1001) and Natural Science Foundation of ShanXi province.

\section{References}

[1] Asm International, Magnesium and Magnesium Alloy [M].OH: Metal Park, 1999. 1

[2] J.E. Gray, B. Luan. Journal of Alloys and Compounds, 2002,336: 88-113.

[3] Chunjiang Ma, Manping Liu, etc. Mater: Sci. Eng. A, 2003, 349: 207-212.

[4] P.M. Jardim, G. Solórzano, J.B. Vander Sande. Mater: Sci. Eng. A, 2004, 381:196-205.

[5] Hong Tae Kang , Terry Ostrom. Mater: Sci. Eng. A, 2008, 490:52-56.

[6] Hinz D, Kirchner A, Brown D, Ma BM, Gutfleisch O. J Mater Process Technol 2003;135:358-3565.

[7] Moshksar M, Ebrahimi R. Int J Mech Sci, 1998,40:1247.

[8] Y.H.Kim, J.H.Park. J. Mater. Proc. Technol., 2003,143-144:735-740

[9] H. HAGHIGHAT, M M. MAHDAVI. Trans. Nonferrous Met. Soc. China, 2013,23:3392-3399

[10]V. Shatermashhadi, B. Manafi, K. Abrinia, etc. Materials and Design, 2014,62:361-366. 
[11]KIM Y H, PARK J H. J Mater Process Technol, 2003, 143-144: 735-740.

[12]M.R.Barnett, Z.Keshavarz, A.G.Beer, D.Atwell. Acta Materialia,2004,52:5093-5103

[13]Qiang Wang, Zhimin Zhang and Xing Zhang. Applied Mechanics and Materials,2012,Vols. 217-219:2304-2307 